\title{
The Effect of Material Processing on the Dielectric Properties of Polystyrene Boron Nitride Nanocomposites
}

\author{
R. Ayoob, T. Andritsch, A.S. Vaughan \\ Tony Davies High Voltage Laboratory \\ University of Southampton \\ Southampton, SO17 1BJ, UK \\ ra1e10@ecs.soton.ac.uk
}

\begin{abstract}
Extensive experimental work in the area of polymer nanocomposites has been done over the past two decades to explore their potential. In this study, a range of related polymer nanocomposite materials was prepared using a solvent blending method, using dichloromethane (DCM), toluene (TOL) and chlorobenzene (CB) to dissolve the polymer, atactic polystyrene (a-PS), and disperse the filler, hexagonal boron nitride (hBN). Where TOL and CB were used, heat was used in material processing, whereas the material was processed at room temperature with DCM. The largest increase in breakdown strength is observed in the materials processed with TOL and CB. The hBN appears to be well dispersed in these systems and more agglomerated in the DCM system as shown from SEM .
\end{abstract}

\section{INTRODUCTION}

The industrial need for enhanced properties of electrical insulation materials has stimulated extensive research into the development of new materials with modified properties [1]. The emergence of a new class of materials termed polymer nanocomposites, or nanodielectrics, has generated considerable interest, as they are frequently reported to exhibit improved electrical properties, such as breakdown strength [2]. While the literature shows consistent results concerning the mechanical properties of these materials, many contradictory findings have been reported regarding electrical properties. This inconsistency in results may be related to variations in material processing techniques used when preparing the polymer nanocomposites. Particular care must be taken during the material preparation process to avoid the issue of nanoparticle agglomeration, which could lead to detrimental material property changes. This study set out to minimise the agglomeration of nanoparticles to achieve a well dispersed system with improved material properties.

A model polymer nanocomposite composed of atactic polystyrene and hexagonal boron nitride is considered in this work. Polystyrene (PS) was chosen due to its amorphous structure, which enables the nanofiller dispersion to be readily imaged, so is ideal for this research. Hexagonal boron nitride (h-BN) was used as the filler due to its relatively high breakdown strength and thermal conductivity [3]. Since the structure of h-BN consists of platelets stacked on top of each other [4], exfoliation of these platelets and dispersing them homogenously within the polymer would theoretically result in the best performance. This study follows our previous work on boron nitride exfoliation [5] and here the choice of solvent to disperse the h-BN was investigated as the solvent is thought to have a considerable effect on the filler dispersion [6].

\section{EXPERIMENTAL}

\section{A. Material Preparation}

The polystyrene was obtained from Sigma-Aldrich with a quoted average molecular mass of $192,000 \mathrm{~g} \mathrm{~mol}^{-1}$. The h-BN was the grade NX-1 obtained from Momentive, with a quoted mean particle size of $900 \mathrm{~nm}$. The materials were prepared using a solution blending procedure, where the solvents used to disperse the h-BN were dichloromethane (DCM), toluene (TOL) and chlorobenzene (CB), as they are all compatible with polystyrene. Isopropyl alcohol (IPA) was used as the nonsolvent to precipitate the polymer/nanofiller mixture. To assess the effect of the choice of solvent, all materials were prepared to have a $5 \mathrm{wt} \%$ of nanofiller and all were sonicated under the same conditions.

The h-BN was first weighed, added to a small amount of the desired solvent and sonicated for half an hour using a probe sonicator. While the h-BN was being sonicated, the polystyrene was added to the required solvent in a separate beaker and stirred using a magnetic stirrer. When DCM was used, the polymer solvent mixture was stirred at room temperature due to the low boiling point of the solvent (i.e. $40{ }^{\circ} \mathrm{C}$ ). However, when toluene and chlorobenzene were used, the polymer solution was stirred at the solvent's boiling point temperature (i.e. at $111^{\circ} \mathrm{C}$ and $131^{\circ} \mathrm{C}$ respectively). After the polymer had dissolved, the sonicated h-BN suspension was added to the polymer solution, stirred, and added to a separate beaker containing IPA to induce precipitation. The resulting nanocomposites were then dried at room temperature in a fume cupboard for 7 days followed by drying in a vacuum oven at $60^{\circ} \mathrm{C}$ for 3 days. The materials were finally melt pressed at $180^{\circ} \mathrm{C}$ with a load of 5 ton. 


\section{B. Differential Scanning Calorimetry}

Differential scanning calorimetry (DSC) traces were obtained from all the samples to examine changes in the glass transition temperature. A Perkin Elmer DSC 7 differential scanning calorimeter operating with Pyris software was used and all experiments were conducted in a nitrogen atmosphere. A sample $\sim 20 \mathrm{mg}$ in mass was placed in an aluminium pan inside the calorimeter and the experiments were performed at a heating rate of $10^{\circ} \mathrm{C} / \mathrm{min}$.

\section{AC Breakdown Strength}

Thin films were prepared for AC breakdown measurements, which were nominally $70 \mu \mathrm{m}$ in thickness. The breakdown strength of these films was measured by placing them between two opposing $6.3 \mathrm{~mm}$ steel ball bearing electrodes immersed in silicone oil to prevent flashover. A $50 \mathrm{~Hz}$ alternating voltage was applied to the films, increasing at a rate of $50 \mathrm{~V} \mathrm{~s}^{-1}$ until breakdown occurred. Twenty breakdown measurements per material were performed and the two-parameter Weibull distribution was used to analyze the data.

\section{Scanning Electron Microscopy}

Samples of $1 \mathrm{~cm} \times 1 \mathrm{~cm} \times 200 \mu \mathrm{m}$ geometry were prepared and etched using a permanganic etching procedure, such that the dispersion of the nanoparticles could be examined. The etching reagent was composed a $1 \%$ solution of potassium permanganate in a mixture composed of 5:2:2 parts sulphuric acid: phosphoric acid: water. The etchant was added to vials containing the samples, and shaken for $4 \mathrm{~h}$. After etching, the samples were quenched in a solution of $75 \%$ composed of $2: 7$ parts sulphuric acid: water plus $\sim 25 \%$ hydrogen peroxide. The samples were then rinsed in distilled water followed by methanol and left to dry for a day before being gold coated. A field emission gun electron microscope (FEG-SEM) JEOL Model JSM-6500F was used to examine the nanoparticle dispersion in all the materials.

\section{RESULTS AND DISCUSSION}

The DSC glass transition temperature $\left(T_{g}\right)$ of all material formulations is shown in Table I. The materials are denoted in the following format: Polymer/nanofiller/wt $\% /$ solvent e.g. $\mathrm{PS} / \mathrm{hBN} / 0 / \mathrm{DCM}$ refers to polystyrene with $0 \mathrm{wt} \%$ of $\mathrm{hBN}$ (i.e. unfilled polystyrene) prepared using DCM and $\mathrm{PS} / \mathrm{hBN} / 5 / \mathrm{DCM}$ refers to a polystyrene boron nitride composite with $5 \mathrm{wt} \%$ filler loading level prepared using DCM. The material denoted PS/Ref refers to reference polystyrene, which was processed directly from pellets which did not undergo any solvent processing. The glass transition temperatures of the two materials prepared using DCM appear to be higher than the materials prepared using TOL and CB. The glass transition temperatures of the materials prepared using TOL and CB are very similar. In all three cases, the glass transition temperature of the nanocomposite is
TABLE I. GLASS TRANSITION TEMPERATURES OF UNFILLED POLYSTYRENE AND COMPOSITES

\begin{tabular}{|l|c|}
\hline \multicolumn{1}{|c|}{ Material } & Glass Transition Temperature $\left({ }^{\circ} \mathbf{C}\right)$ \\
\hline $\mathrm{PS} / \mathrm{Ref}$ & 84.8 \\
\hline $\mathrm{PS} / \mathrm{hBN} / 0 / \mathrm{DCM}$ & 101.7 \\
\hline $\mathrm{PS} / \mathrm{hBN} / 5 / \mathrm{DCM}$ & 101.2 \\
\hline $\mathrm{PS} / \mathrm{hBN} / 0 / \mathrm{TOL}$ & 92.7 \\
\hline $\mathrm{PS} / \mathrm{hBN} / 5 / \mathrm{TOL}$ & 91.6 \\
\hline $\mathrm{PS} / \mathrm{hBN} / 0 / \mathrm{CB}$ & 92.3 \\
\hline $\mathrm{PS} / \mathrm{hBN} / 5 / \mathrm{CB}$ & 91.5 \\
\hline
\end{tabular}

slightly lower than that of the host polymer, when processed in the same way. However, these differences are less than the uncertainties in the data and are therefore statistically insignificant. All samples processed via solution exhibit significantly higher $T_{g}$ values than the reference PS.

Breakdown data were analysed using a Weibull distribution and are presented in Fig. 1. The breakdown strength of the unfilled polystyrene was measured for samples prepared directly from pellets and after solution processing using the three different solvents. The resulting data indicate that the breakdown strength is invariant, which suggests that the imposed drying process results in no residual solvent. Since all the unfilled polystyrene materials exhibit the same breakdown strength, only data from the unprocessed reference material is displayed in Fig. 1 to serve as a reference for the nanocomposite materials. The breakdown strength of the nanocomposite where the h-BN was processed in DCM appears to be similar to that of the host polystyrene. A significant increase in breakdown strength is, however, observed in the nanocomposites prepared using TOL or CB, compared with the unfilled PS. The Weibull scale parameter, $\alpha$, of these two materials is also very similar, just like their glass transition behaviour. We propose that the choice of solvent used has a great impact on the ultimate dispersion of nanoparticles in the system, and that this is the reason for the differences seen in Table I and Fig. 1. The dispersion of the nanoparticles in the polymer was therefore examined and typical results are shown in Fig. 2 and Fig. 3.

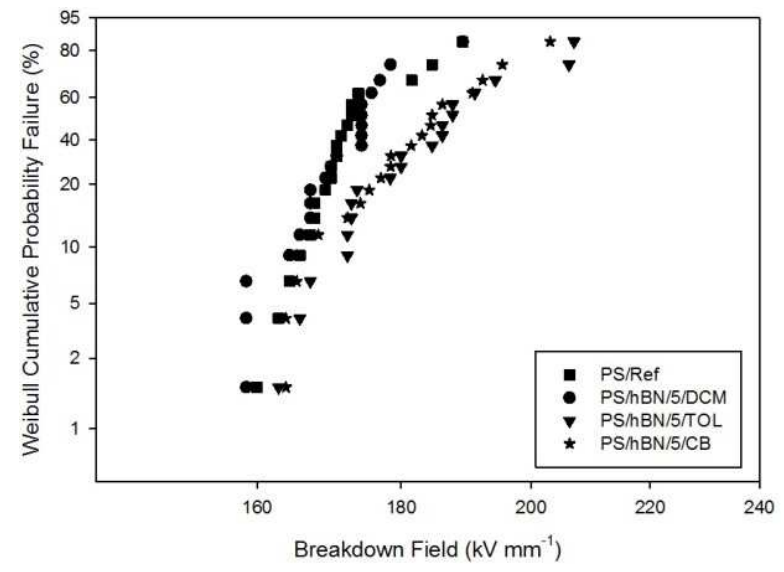

Fig. 1. Weibull Plot of AC breakdown measurements of unprocessed polystyrene and $\mathrm{PS} / \mathrm{hBN}$ composites prepared using different solvents 


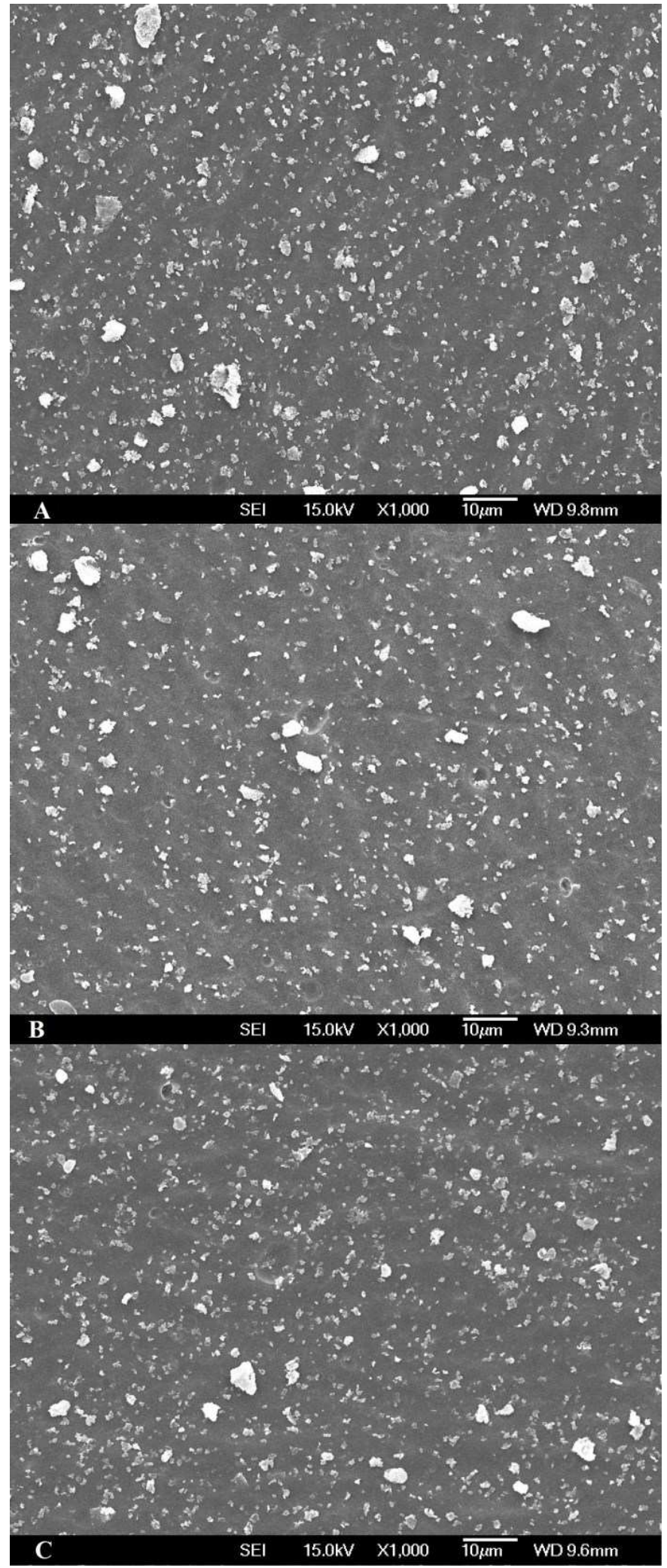

Fig. 2. Low magnification SEM micrographs of (a) PS/hBN/5/DCM, (b) $\mathrm{PS} / \mathrm{hBN} / 5 / \mathrm{TOL}$, (c) $\mathrm{PS} / \mathrm{hBN} / 5 / \mathrm{CB}$

Typical low magnification images of all three materials are shown in Fig. 2. All three of these images reveal that the

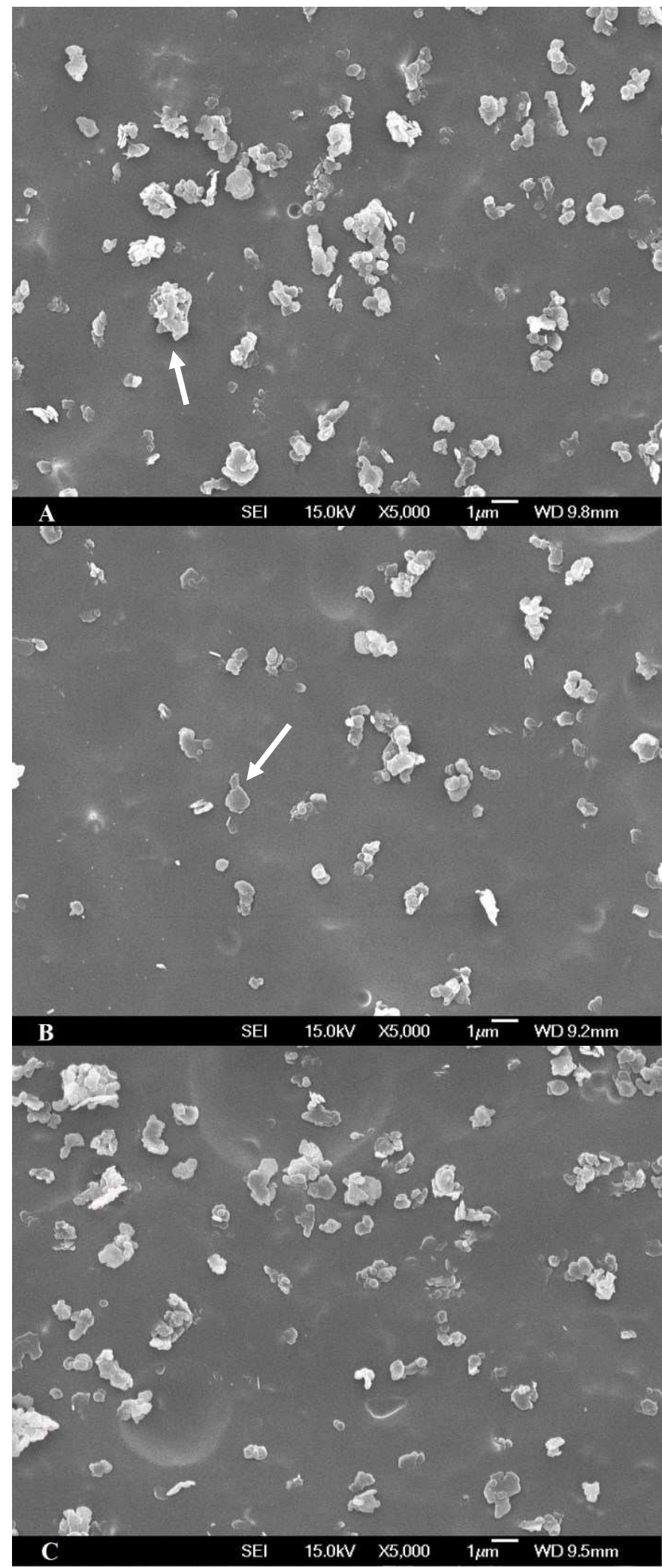

Fig. 3. High magnification SEM micrographs of (a) $\mathrm{PS} / \mathrm{hBN} / 5 / \mathrm{DCM}$, (b) $\mathrm{PS} / \mathrm{hBN} / 5 / \mathrm{TOL}$, (c) $\mathrm{PS} / \mathrm{hBN} / 5 / \mathrm{CB}$

$\mathrm{h}-\mathrm{BN}$ is present in a range of particle sizes, but the number of large agglomerates in Fig. 2a appears somewhat greater than 
in the other two images. Examination of the higher magnification images shown in Fig. 3 reveals that the h-BN exists in various different forms, which can be distinguished by variations in the secondary electron (SE) emission that they generate. In Fig. 3a, the agglomerates typically give rise to relatively high SE emission (arrowed), which indicates that they correspond to regions of high rugosity; this, in turn, implies that they contain many layers of h-BN. In Fig. 3b, many more h-BN objects are present which contain relatively low internal contrast (arrowed). This indicates that the surface is relatively flat and therfore suggests that they contain relatively fewer h-BN layers. In the absence of any supporting data at the moment, we would not refer to these structures as exfoliated h-BN, but we do believe that they are likely to correspond to simple tactoids. Finally, the structure presented in Fig. 3c is intermediate between those discussed above; some extensive agglomerates exist, but tactoids are also present.

The breakdown results are supported by the SEM results in that the materials with the best breakdown performance contain the smaller tactoids. However, the presence of the agglomerates in $\mathrm{PS} / \mathrm{hBN} / 5 / \mathrm{DCM}$ does not adversely affect the breakdown strength as it similar to unfilled material. A possible reason why the $\mathrm{h}-\mathrm{BN}$ is better dispersed in the $\mathrm{PS} / \mathrm{hBN} / 5 / \mathrm{TOL}$ and $\mathrm{PS} / \mathrm{hBN} / 5 / \mathrm{CB}$ systems is that, for both of these solvent systems, elevated termperatures are used during material processing. When the system is heated to the boiling point of the solvent, the system becomes thermodynamically stable at that temperature. When the hot polymer/solvent/nanofiller mixture is poured into the cold IPA non-solvent, this rapid temperature change better entrains the boron nitride within the polymer, even though interactions between h-BN and polystyrene are relatively weak [7].

\section{CONCLUSION}

The thermal analysis data presented in this paper show that the $T_{g}$ of the TOL and CB systems are very similar whereas the DCM system behaves differently. The change in $T_{g}$ in the DCM could be due to the incompatibility between the polymer and solvent or the material processing used where no heat was applied. The data show no observable change in glass transition between nanocomposites and equivalently processed, unfilled polystyrene due to the weak interactions between the filler and the matrix. Electrical analysis using AC breakdown measurements showed that the breakdown strength of the nanocomposites appears to be the highest in the TOL system. The breakdown results of the filled CB system is very close, almost identical, to the filled TOL system. SEM examination of the dispersion state of the h-BN in the various nanocomposites clearly shows that the h-BN is also well dispersed in the $\mathrm{CB}$ system with some agglomerates resulting in a slightly larger average particle size than the TOL system. Finally, the number of agglomerates appear to be the largest in the DCM system suggesting that this processing technique is unfavourable as it is not successful in breaking up the hBN agglomerates and exfoliating the platelets. The tactoids can clearly be seen in the TOL system in Fig. $3 \mathrm{~b}$ which are broken up from the agglomerates, confirming the breakdown results.

From the results, one might conclude that toluene is the best solvent to use in this case. However, these effects might not be the result of the solvent choice alone but rather due to the combined effect of the solvent and the way the material was processed. Since the results for the toluene and chlorobenzene systems were very similar, we might conclude that the similar results might be obtained if another solvent which has a relatively high boiling and is also compatible with polystyrene was used. If this is the case, then the most important factor to test might not be the solvent but the rate at which the gel forms in the material processing procedure so having a large temperature difference might be the key to improving the dispersion in this system.

\section{REFERENCES}

[1] J. G. C. Samuel, M. Fu, S. Lafon-Placette, G. Lucas, and P. J. Howard, "Nano-dielectrics research for application to high voltage insulation systems," 2012 47th Int. Univ. Power Eng. Conf., pp. 1-6, Sep. 2012.

[2] S. Siddabattuni, T. P. Schuman, and F. Dogan, "Improved polymer nanocomposite dielectric breakdown performance through barium titanate to epoxy interface control," Mater. Sci. Eng. B, vol. 176, no. 18, pp. 1422-1429, Nov. 2011.

[3] M. Reading, A. S. Vaughan, and P. L. Lewin, "An investigation into improving the breakdown strength and thermal conduction of an epoxy system using boron nitride," 2011 Annu. Rep. Conf. Electr. Insul. Dielectr. Phenom., pp. 636-639, Oct. 2011.

[4] Y. Lin, T. Williams, and J. Connell, "Soluble, exfoliated hexagonal boron nitride nanosheets," J. Phys. Chem. Lett., vol. 1, no. 1, pp. 277283, Jan. 2010.

[5] R. Ayoob, T. Andritsch, A. S. Vaughan, and Y. Meng, "The Effect of Exfoliation on the Breakdown Strength of Polystyrene Boron Nitride Composites," Annu. Rep. Conf. Electr. Insul. Dielectr. Phenom., pp. 675-678, 2014.

[6] A. B. Morgan and J. D. Harris, "Exfoliated polystyrene-clay nanocomposites synthesized by solvent blending with sonication," Polymer (Guildf), vol. 45, no. 26, pp. 8695-8703, Dec. 2004.

[7] J. Baller, N. Becker, M. Ziehmer, M. Thomassey, B. Zielinski, U. Müller, and R. Sanctuary, "Interactions between silica nanoparticles and an epoxy resin before and during network formation," Polymer (Guildf)., vol. 50, no. 14, pp. 3211-3219, Jul. 2009. 